\title{
Primary cutaneous keratinocytic primordial carcinoma with triple differentiations into basal cell carcinoma, sqaumous cell carcinoma, and porocarcinoma
}

\author{
Tadashi Terada* \\ Department of Pathology, Shizuoka City Shimizu Hospital, Shizuoka, Japan
}

Received: January 4, 2016

Accepted: August 7, 2016

Online Published: August 22, 2016

DOI: $10.5430 /$ crcp.v4n1p7

URL: http://dx.doi.org/10.5430/crcp.v4n1p7

\begin{abstract}
The author herein reports a hitherto undescribed entity of cutaneous primordial carcinoma with triple differentiation into basal cell carcinoma (BCC), squamous cell carcinoma (SCC) and porocarcinoma (PC) in a 66-year-old man. The patient was treated by tumor excision with wide margins. Pathologically, the margins were negative for tumor cells. The patient is now healthy 1 year after the excision. The tumor measured $3 \mathrm{~cm} \times 2 \mathrm{~cm} \times 2 \mathrm{~cm}$ and arose from the epidermis. The percentage of the occupying area was $60 \%$ in BCC, $20 \%$ in SCC, and $20 \%$ in PC. Histologically, BCC was characterized by basalioma cells, melanin pigmentation, peripheral palisading, and cleft formation, SCC by ample acidophilic cytoplasm, individual keratinization and cancer pearls, and PC by poroma cells and tubular structures containing cuticles. Cystic changes in BCC and SCC, keratotic nature of BCC, and acatholytic features of SCC were also seen in places. There were gradual merges among the three components. Immunohistochemically, BCC component was characterized by strong and diffuse expression of KIT, PDGFRA and p63, weak expressions of cytokeratin (CK) AE1/3, CK CAM5.2, CK34BE12, and negative expressions of CK18, CK19, CEA and CA19-9. SCC component was characterized by strongly positive expressions of p63, CEA, CK34BE12, CK7 and CK8, and negative expressions of CK18, CK19, and CA19-9. PC was characterized by strongly positive expressions of KIT, PDGFRA, CK34BE12, CK7, CK8, CK18, CK19, CEA, S100 protein, CA19-9, MUC1, and negative expression of p63. The present case revealed that there are primary cutaneous keratinocytic primordial carcinomas with triple differentiations into BCC, SCC, and PC. The present case is the first example of such tumors. It is thought that KIT and PDFRA-positive stem cells or cancer stem cells in the BCC component give rise to the SCC and PC components in the present case.
\end{abstract}

Key Words: Primary cutaneous tumor, Primordial carcinoma, Basal cell carcinoma, Squamous cell carcinoma, Porocarcinoma

\section{INTRODUCTION}

Many kinds of malignant tumors occur in the skin. They are classified as keratinocytic tumors, melanocytic tumors, appendageal tumors, hematolymphoid tumors, soft tissue tumors, and neural tumor. ${ }^{[1]}$ Of these, the most common is keratinocytic tumors that originate from keratinocytes of the epidermis. They include basal cell carcinoma (BCC), squamous cell carcinoma (SCC), Bowen's disease, actinic

*Correspondence: Tadashi Terada, MD, PhD; Email: piyo0111jp@yahoo.co.jp; Address: Department of Pathology, Shizuoka City Shimizu Hospital, Miyakami 1231 Shimizu-Ku, Shizuoka 424-8636, Japan. 
keratosis, and other malignancies. BCC is defined as a group of malignant cutaneous tumors characterized by the presence of lobules, columns, bands or cords of basaloid cells ("germinative cells"). ${ }^{[2]}$ BCC shows diverse histologies, and characterized by proliferation of basalioma cells, peripheral nuclear palisading, and cleft formations. Many variants of $\mathrm{BCC}$ are present.

SCC of the skin is defined as a malignant neoplasm of the epidermal keratinocytes in which the component cells show variable squamous differentiation. ${ }^{[4]}$ Porocarcinoma (PC), which belongs to skin appendage tumor, ${ }^{[1]}$ is defined as a malignant tumor related to the sweat gland duct, showing intraepidermal and dermal component. ${ }^{[5]} \mathrm{PC}$ is histologically characterized by poroid cells and duct formation. ${ }^{[5]}$

$\mathrm{BCC}$ rarely shows squamous differentiation, and such BCC cases are termed as "basosquamous" carcinoma. BCC also rarely shows adnexal differentiation, and such BCC cases are called "BCC with adnexal differentiation". However, BCC showing both SCC and adnexal differentiation or primordial cutaneous malignancy with triple differentiations into BCC, SCC and adenexal differentiations are not described in the WHO blue book ${ }^{[1]}$ as well as in the world literature, to the best of the author's knowledge.

Herein reported is a case of primary primordial keratinocytic cutaneous carcinoma with triple differentiation into BCC, SCC and PC. A broad immunohistochemical study was performed, and genetic studies of KIT and PDGFRA were performed. The results strongly suggested that the KIT-positive stem cells or cancer stem cells in the BCC component transdifferentiated or differentiated into the SCC and PC components.

\section{CASE REPORT}

A 66-year-old man consulted our hospital because of chest skin tumor. The physical examination revealed a $3 \mathrm{~cm} \times$ $2 \mathrm{~cm} \times 2 \mathrm{~cm}$ tumor with hemorrhage. The clinician's diagnosis was hemangioma, and complete excision with margins was performed. Histologically, the margins were negative for tumor cells and the tumor appeared completely excised without remnant tumor cells. The patient is now free from tumor 1 year after the excision.

Histologically, the tumor measured $3 \mathrm{~cm} \times 2 \mathrm{~cm} \times 2 \mathrm{~cm}$ and arose from the epidermis; the tumor was continuous with the epidermis (see Figure 1A). The tumor was composed of the following three components; BCC (see Figure 1B), SCC (see Figure 1C) and PC (see Figure 1D). The percentage of the occupying area was $60 \%$ in BCC, $20 \%$ in SCC, and $20 \%$ in PC. Histologically, BCC was characterized by basalioma cells, melanin pigmentation, peripheral palisading, and cleft formation, SCC by ample acidophilic cytoplasm, individual keratinization and cancer pearls, and PC by poroma cells and tubular structures containing cuticles. In addition, cystic changes and hemorrhage in BCC and SCC, keratotic nature of BCC, and acatholytic features of SCC were also seen in places. There were gradual transitions or merges among the three components. The post-diagnosis imaging techniques including CT and MRI showed no tumors in the body, thus confirming the safety of the patient, the skin primary nature, and non-metastasizing natures of the tumor.

An immunohistochemical study was performed with the use of Dako Envision method as previously described. ${ }^{[5-7]} \mathrm{Im}$ munohistochemically, the BCC component was characterized by strong and diffuse expression of KIT (see Figure 2A), PDGFRA (see Figure 2B), p63 (see Figure 2C), weak expressions of cytokeratin (CK) AE1/3, CK CAM5.2, CK34BE12, and negative expressions of CK18, CK19, CEA and CA19-9. SCC component was characterized by strongly positive expressions of p63, CEA, CK34BE12, CK7 (see Figure 2D), and CK8, and negative expressions of CK18 (see Figure 2E), CK19, and CA19-9. PC was characterized by strongly positive expressions of CK34BE12, CK7, CK8, CK18 (see Figure 2E), CK19, CEA, S100 protein, CA19-9, MUC1, KIT (see Figure 2F), and PDGFRA, and negative expression of p63. In all of the BCC, SCC, and PC components, strong and diffuse expressions of p53 and Ki-67 with high labeling indexes were seen.

Because the BCC and PC showed strong expression of KIT and PDGFRA, a molecular genetic analysis of KIT gene (exons 9, 11, 13, and 17) and PDGFRA gene (exons 12 and 18) was performed in the cutaneous tumor by the PCR direct sequencing method, as previously reported. ${ }^{[8-13]}$ The tissues of two GISTs and a small cell lung carcinoma (SCLC) were used as positive and negative controls, respectively; the GIST cases had been detected to be positive for $\mathrm{KIT}^{[8]}$ or PDGFRA mutation $^{[9]}$ and the SCLC to be negative for KIT mutation. The separation of each tissue was performed by laser-captured microdissection (LCM) from formalin-fixed paraffin-embedded (HFPE) tissue, and DNA of each tissue element was extracted by ethanol sedimentation. The molecular analysis revealed no mutations of genes of KIT (exons 9, 11, 13, and 17) and PDGFRA (exons 12 and 18) genes in this cutaneous tumor, and imatinib may be ineffective. The positive control and negative control showed mutations of KIT or PDGFRA (positive controls) and no mutation (negative control).

\section{Discussion}

The present case revealed that there are primary cutaneous keratinocytic primordial carcinomas with triple differenti- 
ations into BCC, sqaumous cell carcinoma, and porocarcinoma. The present case is the first one of such tumors.

The most important findings to understand this peculiar tumor are positive KIT and positive PDGFRA in the BCC component. AS is well known, KIT and platelet-derived growth factor receptor- $\alpha$ (PDGFRA) genes, both mapped to 4q12, encode transmembranous receptor tyrosine kinase oncoproteins called KIT (CD117) and PDGFRA, respectively. ${ }^{[8-14]}$ Both molecules are transmembranous oncoproteins involved in tumorigenesis, particularly in GIST, e-GIST, malignant melanoma, germ cell tumors, hematopoietic malignancies, small cell carcinoma, large cell neuroendocrine carcinoma, adenoid cystic carcinoma, myoepithelial carcinoma, BCC, and other tumors. In these cancers, KIT and PDGFRA are
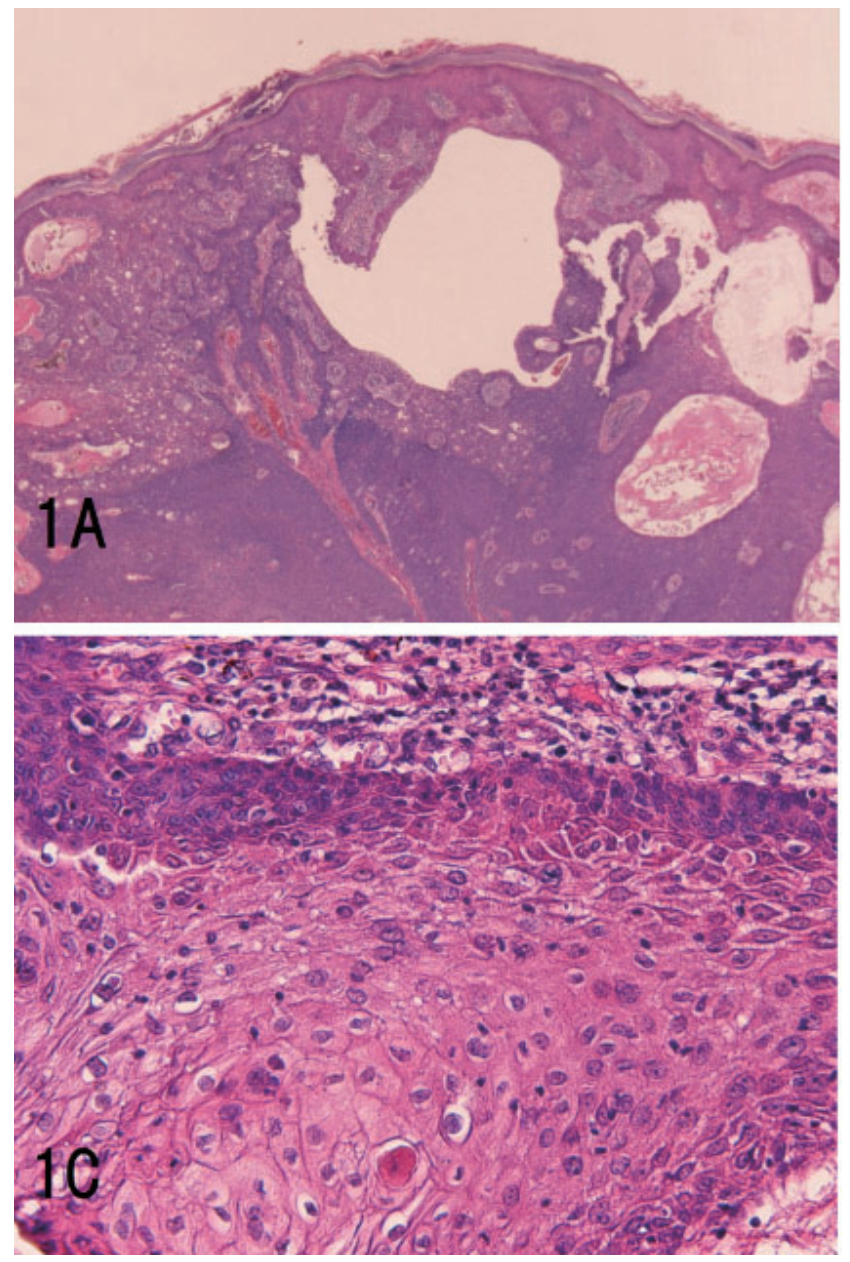

known to be expressed in cancer cell membranes. In embryonic biology, KIT and PDGFRA are expressed in embryonic stem cells. Probably, PDGFRA is expressed in cancer stem cells. In the present tumor, the major component is BCC that were strongly and diffusely positive for KIT and PDGFRA. Thus, it seems very probable in the present tumor that KITpositive and PDGFRA-positive stem cells or cancer stem cells in the BCC component transdifferentiated or differentiated into the SCC and PC components, thus creating this primary cutaneous keratinocytic primordial carcinoma with triple differentiations into BCC, sqaumous cell carcinoma, and porocarcinoma. Histologically, there were gradual transitions and merges among the three components suggest the above comments.
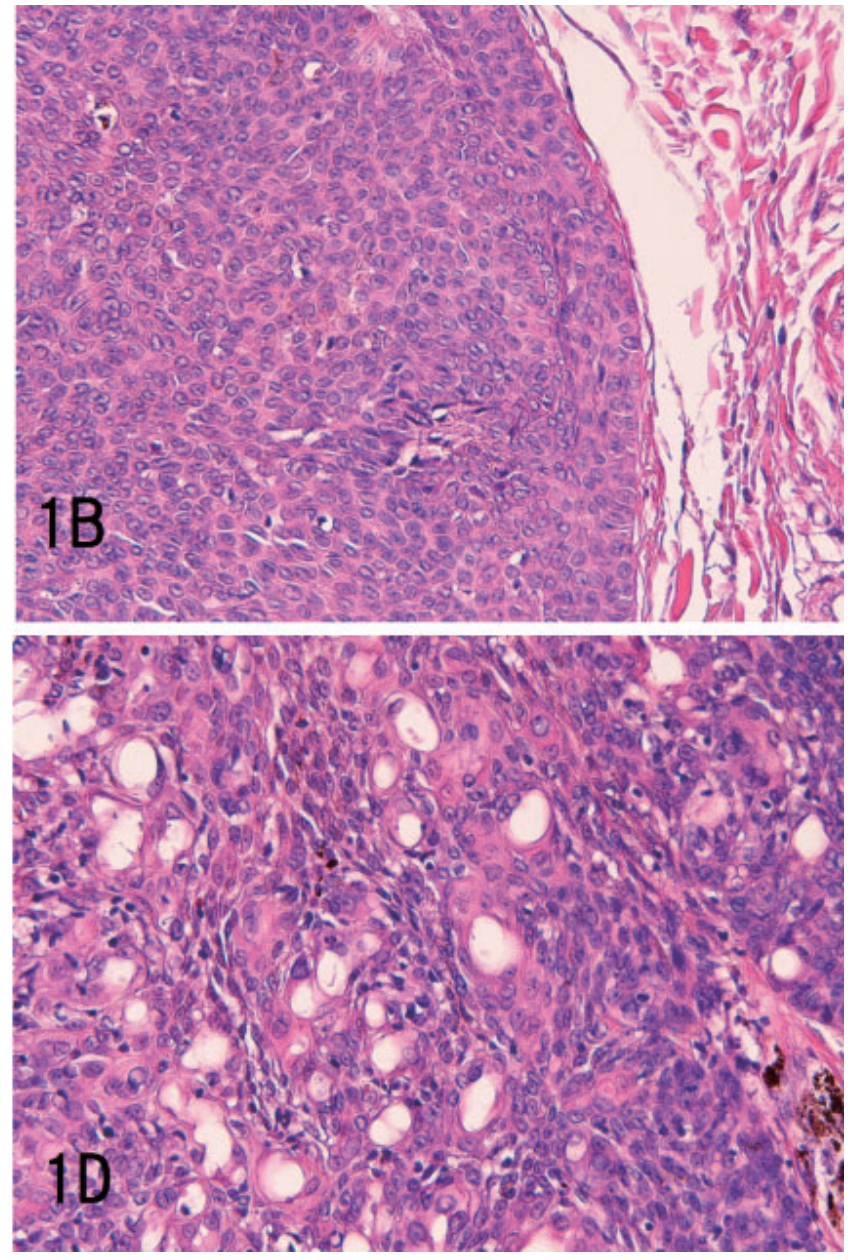

Figure 1. Histologic features

A: Low power view. The tumor originates from the epidermis. Cystic and hemorrhagic, acantholytic changes are apparent. The three components of basal cell carcinoma $(B C C)$, squamous cell carcinoma $(B C C)$ and porocarcinoma $(P C)$ are apparent. HE, $\times 20$. B: High power view of BCC component. The basalioma cells, peripheral palisading, and cleft formations are obvious. $H E, \times 200$. C: High power view of SCC component. The atypical features, kerainization and intercellular bridges are apparent. HE, $\times 200$. D: High power view of PC component. The malignant poroma cells and tubules formations with outer cuticle-like basal cells are obvious. $H E, \times 200$. 
The present study showed no mutations of the KIT and PDGFRA genes in their hot spots. Among various KITor PDGFRA-positive tumors, the mutations of both genes are recognized in GIST, e-GIST, and hematopoietic malignancies, germ cell tumors, and melanomas. Other positive tumors such as small cell carcinomas, adenoid cystic carcinomas, neuroendocrine tumor and carcinomas, mast cell neoplasms, breast carcinomas, usually lack mutations of the KIT and PDGFRA in their hot spots. Mutational analysis of all exons and introns of the both genes is a future problem.
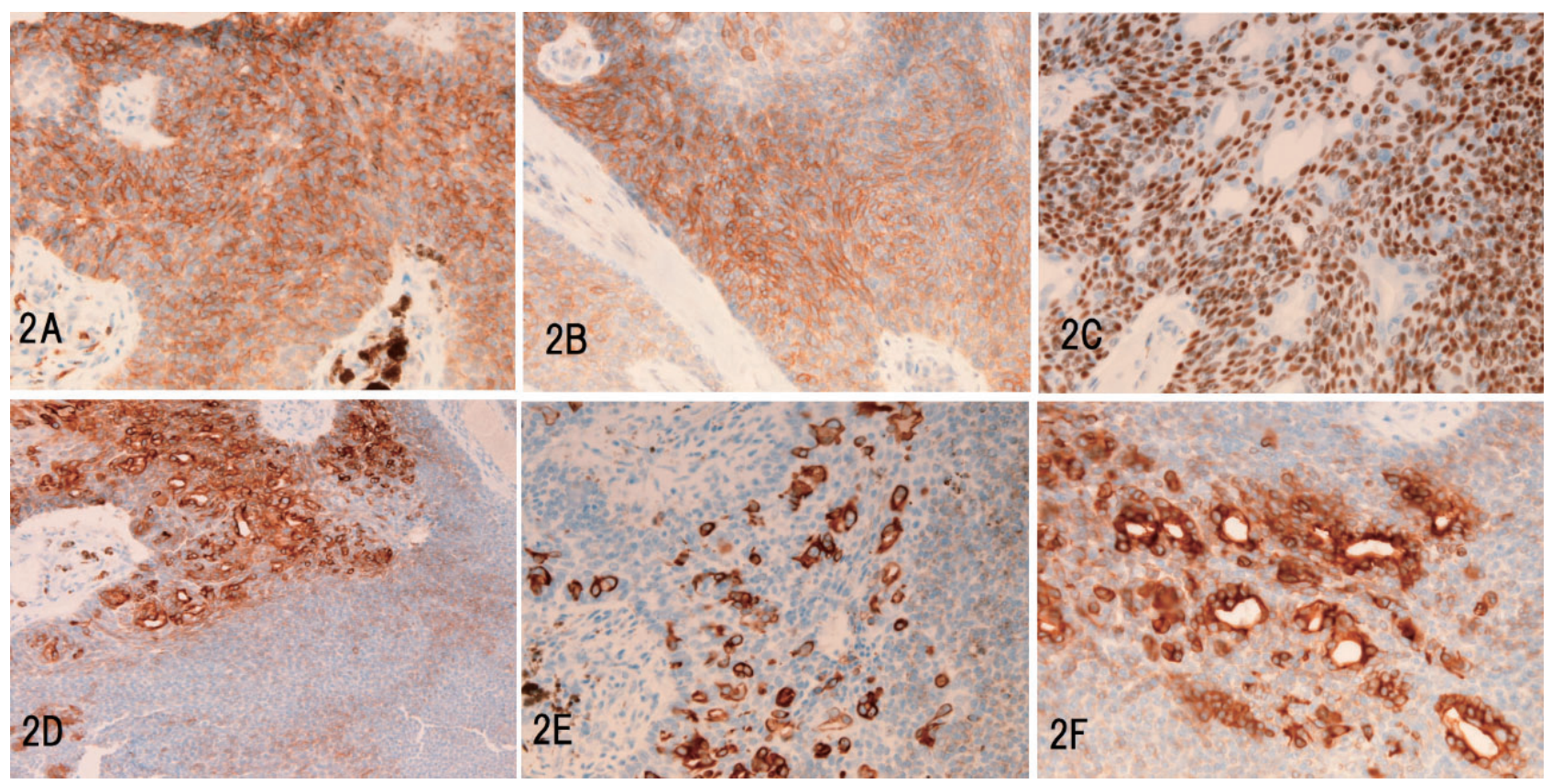

Figure 2. Immunohistochemical features

A: Strong and diffuse expression of KIT is seen in basal cell carcinoma (BCC) component. $\times 200$. B: The strong and diffuse expression of PDGFRA in the BCC component is evident. $\times 200$. C: There is a strong and diffuse expression p63 in the BCC and squamous cell carcinoma (SCC) components. $\times 200$. D: Cytokeratin (CK) 7 is strongly expressed in the SCC and porocarcinoma (PC) components (upper), but is negative or is faintly expressed in BCC component (lower). E: CK 18 is expressed in the PC component, but not in the $B C C$ and SCC components. $\times 200$. F: The PC component strongly and diffusely expresses KIT. $\times 200$.

The author's previously works ${ }^{[14]}$ using 66 case of pure BCC showed that pure BCC very frequently expressed KIT, PDGFRA and neuroendocrine antigens (NCAM, NSE, synaptophysin and chromogranin). The NCAM, NSE, synaptophysin and chromogranin are antigens of stem cells in admission to KIT and PDGFRA. Thus, pure BCC seems to have characters of stem cells. In the present case, there were no expressions of synaptophysin, chromogranin, NCAM and NSE, suggesting that the present tumor shows little neuroendocrine differentiation. However, since the present tumor was strongly and diffusely positive for KIT and PDGFRA in the $\mathrm{BCC}$ and PC components, the stem cell nature of the present case seems to be certainly present. In the previous study of pure BCC, the author did not examine genes of KIT and PDGFRA because the materials were too many. In the present tumor, the author can investigated the molecular study.

The pathological diagnosis of the present tumor seems valid. The BCC component is composed of basalioma cells, and characteristic findings of BCC such as peripheral nuclear palisading, cleft formation, stromal myxoid changes, melanin deposition were recognized. These features apparently fulfill the diagnostic criteria of BCC. ${ }^{[2]}$ The SCC component showed ample eosinophilic cytoplasm, malignant features, individual keratinizations, and cancer pearls, thus fulfilling the WHO criteria of skin SCC. ${ }^{[3]}$ The PC component was composed of malignant poroid cells and scattered tubular structures with outer curticle-like substances that are similar to intraepidermal sweat (eccrine) glands. These features fulfill the criteria of $\mathrm{PC}$ of $\mathrm{WHO} .^{[4]}$

BCC infrequently expresses features of SCC and appendage tumors, and such tumors are termed as "basosquamous" carcinoma or "basaloid SCC", for the former two and "BCC with adnexal differentiation" for the last, respectively. However, there has been no descriptions of the primordial cutaneous carcinoma with triple differentiations into BCC, SCC and $\mathrm{PC}$, as seen in the present study, in the text books including WHO and in the world literature, to the best of the author's 
knowledge. Thus, the present tumor seems to pose a new entity.

In the present tumor, many immunohistochemical investigations were performed. Although the author wants to mention many comments, the discussion is deduced into minimum because of space limitation. Immunohistochemically, BCC component was characterized by strong and diffuse expression of KIT, PDGFRA and p63, weak expressions of CK AE1/3, CK CAM5.2, CK34BE12, and negative expressions of CK18, CK19, CEA and CA19-9. P63 is a maker of BCC, SCC, and myoepithelial cells and their malignant counterparts. $\mathrm{P} 63$ was positive in the present $\mathrm{BCC}$ component, being compatible with BCC. The cytokeratin profile in BCC component showed predominant high-molecular-weight (MW) CKs. The CD7+/CK20- pattern is compatible with primary cutaneous BCC. CK14 is known to be expressed in adenoid cystic carcinoma and BCC. ${ }^{[14]}$ The negative CK18 and CK19 indicate the BCC component does not have low MW CK. The negative CEA and CA19-9 indicate that the BCC component has not glandular phenotypes.

The SCC component was characterized by strongly positive expressions of p63, CEA, CK34BE12, CK7 and CK8, and negative expressions of CK18, CK19, and CA19-9. In this respect, comments similar to those of $\mathrm{BCC}$ are made. Characteristically, the SCC component was negative for KIT and PDGFRA, suggesting they have no stem cells, or transdifferentiated cells losing KIT and PSGDRA from the BCC component. The expression of CEA in the SCC component is very curious, and may reflect that this SCC component is different from ordinary SCC but has glandular phenotypes.

PC was characterized by strongly positive expressions of KIT, PDGFRA, CK34BE12, CK7, CK8, CK18, CK19, CEA, S100 protein, CA19-9, MUC1, and negative expression of p63. The significant of PIT and PDGFRA were already described. The CK profile shows wide ranges of CK from low-MW and high MW CKs in contrast with BCC and SCC components which showed dominant high molecular weight CK. The CK 18 and CK19 are well markers of eccrine differentiation, since these two CKs were never expressed in

\section{REFERENCES}

[1] LeBoit PE, Burg G, Weedon D, et al. World Health Organization Classification of tumours. Pathology and genetics of skin tumours. Lyon: IARC Press; 2006.

[2] Kossard S, Epstain Jr. EH, Cerio R, et al. Basal cell carcinoma. In: LeBoit PE, Burg G, Weedon D, Sarasin A eds. World Health Orga-
BCC and SCC in the present study. The positive CK18 and CK19 Characteristically CEA, CA19-9, and MUC were positive, indicating the PC component shows really eccrine gland phenotype. The negative expression of p63 may shows this component is not $\mathrm{BCC}$ and SCC. The positive $\mathrm{S} 100$ protein and CK14, which are antigens of myoepithelial and basal cells, suggest the myoepithelial duct phenotypes and is very compatible with PC.

In all the three components, the p53 was strongly and broadly positive, suggesting the $\mathrm{p} 53$ gene mutations and malignant potential of the present study. The Ki-67 labeling was also high in all the three components, reflecting high cellular proliferation and malignant potentials.

Biologically, the present tumor seems to behave like BCC because the dominant component was $\mathrm{BCC}$, and the other two components were not widely invasive and have no significant atypia. Therefore, the prognosis of the present tumor seems excellent.

Finally, quite interestingly several cell growth signaling molecules were seen in the present case. These findings suggest that SCF/KIT, PDGFa/PDGFRA, EGF/ErbB2, and HGF/MET signaling pathways play an important roles in the development and progression of the present tumor.

\section{Conclusions}

The author presented a peculiar tumor of the skin of primary cutaneous keratinocytic primordial carcinoma with triple differentiations into BCC, sqaumous cell cartinoma, and porocarcinoma. The histological findings and immunohistochemical findings of KIT and PDGFRA, stem cell antigens, suggested that the cancer stem cells within BCC component differentiated into SCC and porocarcinoma components in this primordial carcinoma with triple differentiations. There were no mutations in the KIT and PDGFRA genes in molecular studies. The present case is the first report of such triple differentiation tumor. An extensive immunohistochemical study was performed.

\section{CONFLiCTS OF INTEREST Disclosure}

The author declares no conflicts of interest. nization Classification of tumours. Pathology and genetics of skin tumours. Lyon: IARC Press; 2006. 13p.

[3] Weedon D, Morgan MB, Gross C, et al. Squamous cell carcinoma; In: LeBoit PE, Burg G, Weedon D, Sarasin A eds. World Health Organization Classification of tumours. Pathology and genetics of skin tumours. Lyon: IARC Press; 2006. 20p. 
[4] Requena L, Mengesha YM, Kulzner H. Malignant tumors with apocrine and eccrine differentiation. Porocarcinoma. In: LeBoit PE, Burg G, Weedon D, Sarasin A eds. World Health Organization Classification of tumours. Pathology and genetics of skin tumours. Lyon: IARC Press; 2006. 128p.

[5] Terada T, Kawaguchi M, Furukawa K, et al. Minute mixed ductalendocrine carcinoma of the pancreas with predominant intraductal growth. Pathol Int. 2002; 52: 740-6. PMid: 12685552. http: //dx.doi.org/10.1046/j.1440-1827.2002.01416.x

[6] Terada T, Takeuchi T, Taniguchi M. Hepatobiliary cystadenocarcinoma with cystadenoma elements of the gall bladder in an old man Pathol Int. 2003; 53: 790-5. PMid: 14629305. http://dx.doi.o $\mathrm{rg} / 10.1046 / \mathrm{j} .1440-1827.2003 .01559 . \mathrm{x}$

[7] Terada T, Tanigichi M. Intraductal oncocytic papillary neoplasm of the liver. Pathol Int. 2004; 54: 116-23. PMid: 14720143. http: //dx.doi.org/10.1111/j.1440-1827.2004.01594.x

[8] Terada T. Primary multiple extragastrointestinal stromal tumors of the omentum with different mutations of c-kit gene. World J Gastroenterol. 2008; 14: 7256-9. PMid: 19084944. http://dx.doi.o $\mathrm{rg} / 10.3748 / \mathrm{wjg} \cdot 14.7256$

[9] Terada T. Primary extragastrointestinal stromal tumor of the transverse mesocolon without c-kit mutations but with PDGFRA mu- tations. Med Oncol. 2009; 26: 233-7. PMid: 18777214. http: //dx.doi.org/10.1007/s12032-008-9092-9

[10] Terada T. Autopsy case of primary small cell carcinoma of the urinary bladder: KIT and PDGFRA expression and mutations. Pathol Int. 2009; 59: 247-50. PMid: 19351368. http://dx.doi.org/10.11 11/j.1440-1827.2009.02358.x

[11] Terada T. Small cell neuroendocrine carcinoma of the prostate: Incidence and a report of four cases with an examination of KIT and PDGFRA. Prostate. 2012; 72: 1150-6. PMid: 22127977. http: //dx.doi.org/10.1002/pros. 22464

[12] Terada T. Protein expression and gene mutation status of KIT and PDGFRA in renal cell carcinoma. Histol Histopathol. 2012; 27: 297302. PMid: 22237707.

[13] Terada T. Mutations and protein expression of KIT and PDGFRA genes in ipsilateral testicular seminomas: an immunohistochemical and molecular genetic study. Appl Immunohistochem Mol Morphol 2011; 19: 450-3. PMid: 21403518. http://dx.doi.org/10.10 97/PAI . Ob013e31820d2872

[14] Terada T. Expression of NCAM (CD56), chromogranin A, synaptophysin, c-KIT (CD117) and PDGFRA in normal non-neoplastic skin and basal cell carcinoma: an immunohistochemical study of 66 consecutive cases. Med Oncol. 2013; 33: 444. PMid: 23292839. http://dx.doi.org/10.1007/s12032-012-0444-0 